\title{
Effect of Functional Groups on Incorporation Behavior of Amino Acid-Type Surfactant into Phospholipid Vesicle Membrane
}

\author{
Akio Ohta $^{1 *}$, Yuya Miyazato ${ }^{1}$, Hiroyasu Sasaki ${ }^{1}$, Kazuma Yasuhara $^{2}$ and \\ Tsuyoshi Asakawa ${ }^{1}$ \\ ${ }^{1}$ School of Chemistry, College of Science and Engineering, Kanazawa University (Kakuma, Kanazawa, Ishikawa 920-1192, JAPAN) \\ ${ }^{2}$ Graduate School of Material Science, Nara Institute of Science and Technology (8916-5, Takayama-cho, Ikoma, Nara 630-0192, JAPAN)
}

\begin{abstract}
The incorporation behaviors of some $N$-dodecanoyl amino acid-type surfactants into L- $\alpha$ dipalmitoylphosphatidylcholine (DPPC) vesicles in aqueous solution were investigated. From the leakage measurements of a vesicle-entrapped fluorescence probe, it was found that these surfactants did not affect the DPPC vesicle so much at a very low concentration less than a one-tenth of the critical micelle concentration (CMC), but caused a significant release of the probe form the vesicles even at just below the CMC. The leakage induced by amino acid-type surfactants was promoted by increasing hydrophobicity of the amino acid. However the polarization of DPH embedded in the DPPC membrane was almost unchanged by incorporation of the surfactants at the concentration below the CMC except for the $\mathrm{N}$ dodecanoylphenylalanine system. The binding enthalpy and the binding constant of the surfactant to DPPC vesicles were estimated by isothermal titration calorimetry. While the binding enthalpy was independent of the kind of surfactant, the binding constant increases with increasing hydrophobicity of the amino acid species in the surfactants. The molar ratio of bound surfactant to DPPC molecule in the saturated state was much greater than that for sodium dodecyl sulfate system. Further the ratio was not influenced by the structure of the amino acid side chain.
\end{abstract}

Key words: $N$-acyl amino acid-type surfactant, phospholipid, vesicle, fluorescence probe, ITC

\section{INTRODUCTION}

Phospholipid is the main component of biological cell membrane. The interaction between phospholipid vesicle membrane and surfactant is of great interest from the standpoints of the solubilization and the reconstitution of membrane proteins, and hence, many researchers have reviewed about it ${ }^{1-6)}$. In general, the nonionic surfactants have been widely used for this purpose ${ }^{7-17)}$. On the other hand, anionic surfactants are used as detergents, and then the interaction of anionic surfactants with phospholipid vesicles might be a good research model for the influence of detergent on skin ${ }^{18-21)}$. Particularly $N^{-}$ acyl amino acids, which are anionic amino acid-type surfactants, are very valuable because of their biodegradability and low toxicity ${ }^{22,23}$. In spite of the interest in their low toxicity, there are few studies on the interaction between amino acid-type surfactants and phospholipid vesicles from the standpoint of their physicochemical properties, except for those concerning their antimicrobial activity ${ }^{24-26)}$.

In our previous study, the incorporation behaviors of sodium $N$-dodecanoyl-sarcosinate and -L-glutamate into phospholipid vesicle were examined, and the results were compared with those of sodium dodecyl sulfate (SDS) and sodium dodecanesulfonate ${ }^{27)}$. This study revealed the unique behaviors of amino acid-type surfactants; i.e., the cooperative adsorption on the vesicle membrane occurs for amino acid-type surfactant systems, and these surfactant molecules are organized on the vesicle. Therefore, in this work, the incorporation behavior of some amino acid-type surfactants into DPPC vesicles was investigated systematically for the purpose of an elucidation of the effect of amino acid side chain. We chose the following four amino

*Correspondence to: Akio Ohta, School of Chemistry, College of Science and Engineering, Kanazawa University , Kakuma, Kanazawa, Ishikawa 920-1192, JAPAN

E-mail: akio-o@t.kanazawa-u.ac.jp

Accepted August 24, 2009 (received for review July 18, 2009)

Journal of Oleo Science ISSN 1345-8957 print / ISSN 1347-3352 online

http://www.jstage.jst.go.jp/browse/jos/ 


\section{A. Ohta, Y. Miyazato, H. Sasaki et al.}

acid surfactants, sodium $N$-dodecanoyl-glycinate (C12Gly), -L-valinate (C12Val), -L-leucinate (C12Leu), and-L-phenylalaninate (C12Phe).

It is known that the critical micelle concentration (CMC) of the amino acid surfactants decreases monotonously with increasing hydrophobicity of the amino acid side chain without exception ${ }^{28}$. The negative enthalpy of micelle formation and high Krafft temperature is characteristic of $\mathrm{C} 12 \mathrm{Phe}$. Although these phenomena could not be explained in terms of only the hydrophobicity of the side chain ${ }^{28,29)}$, these results suggest the presence of a strong interaction between the side chains of phenylalanine in the micelle or hydrated solid states. In such a way, the side chain of $\mathrm{N}$-acyl amino acid surfactants greatly influences their physical properties. Thus, it is important to investigate the effect of the amino acid side chain on the incorporation behavior of these surfactants into DPPC vesicle membranes. This was done mainly by the fluorescence measurements for both the release of a vesicle-entrapped probe and the polarization of DPH in lipid membrane in the presence of a series of surfactant as a function of the surfactant concentration. In addition, the binding constant and the binding enthalpy of the surfactant to DPPC vesicles were estimated by isothermal titration calorimetry (ITC).

\section{EXPERIMENTAL}

\subsection{Materials}

Sodium $N$-dodecanoyl glycinate (C12Gly), sodium $N$ dodecanoyl L-valinate (C12Val), sodium $N$-dodecanoyl Lleucinate (C12Leu), and sodium $N$-dodecanoyl L-phenylalaninate (C12Phe) were prepared from respective $\mathrm{N}$-dodecanoyl amino acids by addition of an equivalent quantity of sodium hydroxide. All the above substances were purified by recrystallization from ethanol solution. Except for C12Gly, L-amino acids were employed as an ingredient. DPPC and 5(6)-carboxy fluorescein (CF) were purchased from Wako Pure Chemical Industries Ltd. and Kanto Chemical Co. Inc., respectively, and were used as received. In this experiment, $\mathrm{CF}$ was used as a mono-sodium salt. For the preparation of vesicular suspensions, $0.1 \mathrm{M}$ tris(hydroxymethyl)- aminomethane (tris)- $\mathrm{HCl}$ buffer at $\mathrm{pH}$ $=7.4$ was employed. 1,6-Diphenyl-1,3,5-hexatriene (DPH) was used as a fluorescence probe for the vesicle membrane, which was purchased from Aldrich.

Vesicle suspensions were prepared by hydration of a dry DPPC film (20 mg) with $2 \mathrm{~mL}$ of tris- $\mathrm{HCl}$ buffer or that with CF $(0.1 \mathrm{M})$. Finally, the vesicle suspensions were extruded into $100 \mathrm{~nm}$ polycarbonate filter on an extruder (Avanti Polar Lipids Mini-Extruder) at $320 \mathrm{~K}^{30}$. The $\mathrm{CF}$ encapsulating vesicles were separated from untrapped $\mathrm{CF}$ molecules by gel filtration. The concentration of DPPC was confirmed by determining the quantity of the choline group using a special assay kit from Wako Pure Chemicals ${ }^{31}$.

\subsection{Surface tension measurements}

The surface tension of the surfactant solution was measured at $298.2 \mathrm{~K}$ using the Wilhelmy glass plate method with a Kyowa EBS-V tensiometer (Saitama, Japan) with an accuracy of $\pm 0.2 \mathrm{mNm}^{-1}$.

\subsection{Leakage Measurements}

About ten microliters of vesicle suspension were added to $1 \mathrm{~mL}$ portions of several surfactant solutions so that the concentration of DPPC was $0.1 \mathrm{mM}$. Fluorescence measurements were performed using a Hitachi F-3010 fluorescence spectrometer at $298.2 \mathrm{~K}$. The fluorescence intensity of leaked CF was measured at $515 \mathrm{~nm}$ by excitation at 490 $\mathrm{nm}$. Excitation and emission band-passes were $1.5 \mathrm{~nm}$. At the end of the measurements, $100 \mu \mathrm{L}$ of $10 \mathrm{wt} \%$ Triton X100 solution was added to all fractions in order to rupture all of the vesicles, and the fluorescence intensity was measured again.

\subsection{Polarization measurements}

The polarization $P$ defined by

$$
P=\frac{I_{v v}-G I_{v h}}{I_{v v}+G I_{v h}}
$$

was obtained from the fluorescence intensity of DPH by using a Perkin Elmer LS55 spectrometer. Here $G$ is the grating factor, which is calculated by the following equation

$$
G=I_{h v} / I_{h h},
$$

where $I_{h v}$ is the intensity with horizontal and vertical polarizers (excitation and emission, respectively), and $I_{h h}$ is the intensity with horizontal polarizers for both emission and excitation. The fluorescence intensity of DPH was measured at $428 \mathrm{~nm}$ by excitation at $357 \mathrm{~nm}$. It has been reported that DPH is oriented to the lipid membrane ${ }^{32)}$. Therefore, the molecular motion is restricted at high viscosity and the $P$ value reflects the fluidity at the hydrophobic core of the molecular aggregates. DPH molecules were added to methanol solution of DPPC, and then dry DPPC film with DPH was prepared. The ratio of DPPC to DPH was $1000: 1$ and the final concentration of DPH was $10^{-7}$ $\mathrm{M}$, whereas DPPC concentration in each sample was 0.1 $\mathrm{mM}$.

\subsection{Calorimetry}

The enthalpy of dilution of a concentrated solution of the surfactant was measured by isothermal titration microcalorimetry (MCS-ITC, MicroCal, Northampton, MA) at $298.15 \mathrm{~K}$. Ten microliters of the aqueous solution of each 
surfactant at ca. 15-fold concentration of their CMC was injected using a computer-controlled syringe pump into 1.5 $\mathrm{mL}$ of tris- $\mathrm{HCl}$ buffer solution or DPPC suspension in the cell. DPPC concentration was varied from 0.02 to $0.14 \mathrm{mM}$.

\section{RESULTS and DISCUSSION}

\subsection{Critical Micelle Concentrations by Surface Tension Measurements}

The surface tension $\gamma$ of the aqueous solutions of several surfactants was shown in Fig. 1 as a function of molarity $c_{S}$ of the surfactant at $298.2 \mathrm{~K}$. The $\gamma$ values of the aqueous solution in the presence of $0.1 \mathrm{mM} \mathrm{DPPC}$ were over-plotted in respective figures. The $\gamma$ values decrease steeply with increasing $c_{\mathrm{S}}$ and all curves have a break point at CMC. It is seen that the $\gamma$ values of the solutions of C12Gly and C12Leu are not changed even though the DPPC vesicles coexist. In contrast, the $\gamma$ value for $\mathrm{C} 12 \mathrm{Phe}$ system significantly increased in the range of $0.1-0.6 \mathrm{mM}$ by the addition of DPPC vesicles following increase of CMC. Furthermore we observed another break point at lower concentration in the curve of $\mathrm{C} 12 \mathrm{Phe}$ with DPPC vesicle system. It is suggested that, in the region between two breakpoints, the C12Phe molecules are highly accumulated on the DPPC vesicle membrane below the $\mathrm{CMC}$, thus, its concentration in bulk phase reduced. On the other hand, both surface tension of aqueous solution and the CMC values of SDS slightly decreased in the presence of DPPC vesicles. This fact suggests that SDS molecules affect the DPPC vesicles below the CMC to induce the spontaneous formation of mixed monolayer of SDS and DPPC at the air/water interface $^{33)}$.

\subsection{Leakage and Fluidity of Vesicle Membrane}

The values of the leakage ratio of $\mathrm{CF}$ molecules, which were estimated from the fluorescence intensity of the samples, were plotted in Fig.2 as a function of time for the four amino acid-type surfactants at various concentrations, $C *\left(=c_{S} / \mathrm{CMC}: \mathrm{CMC}\right.$ is obtained by surface tension measurement with DPPC). The leakage ratio $L$ was defined by

$$
L(\%)=\frac{I-I_{\mathrm{bg}}}{I_{\text {triton }} \times r-I_{\mathrm{bg}}} \times 100
$$

where $I$ and $I_{\text {triton }}$ are the fluorescence intensity of the samples during the measurements and at the end of the measurements when all vesicles were completely ruptured by Triton X-100, respectively, and $r$ is the dilution factor due to the addition of $100 \mu \mathrm{L}$ of $10 \mathrm{wt} \%$ Triton X-100 solution; in this case, the $r$ value was 1.1. The background intensity $I_{\mathrm{bg}}$ was estimated by

$$
I_{\text {bg }}=I_{\text {triton }} \times \frac{I_{\text {buffer }}}{I_{\text {triton+buffer }}}
$$

where $I_{\text {buffer }}$ is the fluorescence intensity of the surfactantfree sample and $I_{\text {triton+buffer }}$ is that of the mixture of the sample and Triton X-100. For all the systems, the leakage of CF from the vesicles was hardly induced by the addition of the surfactants below $C^{*}=0.02$ ( Fig.2(a)). On the other hand, above $C^{*}=1$ the encapsulated $\mathrm{CF}$ was released spontaneously except for C12Gly system. At intermediate concentration, $C^{*}=0.2($ Fig.2(b)), the leakage kinetics are different depending on the type of surfactants. The initial rate of dye leakage increased with increasing the hydrophobicity of amino acid.

The values of the leakage ratio at $2 \mathrm{~h}$ after the mixing were also plotted against the reduced concentration $C *$ in Fig.3 for the four surfactant systems. Above $C^{*}=1$, the release of $\mathrm{CF}$ molecules was completed for all systems. This result shows that these surfactant molecules ruptured the DPPC vesicles to form the mixed micelles. However the encapsulated CF in DPPC vesicles was already
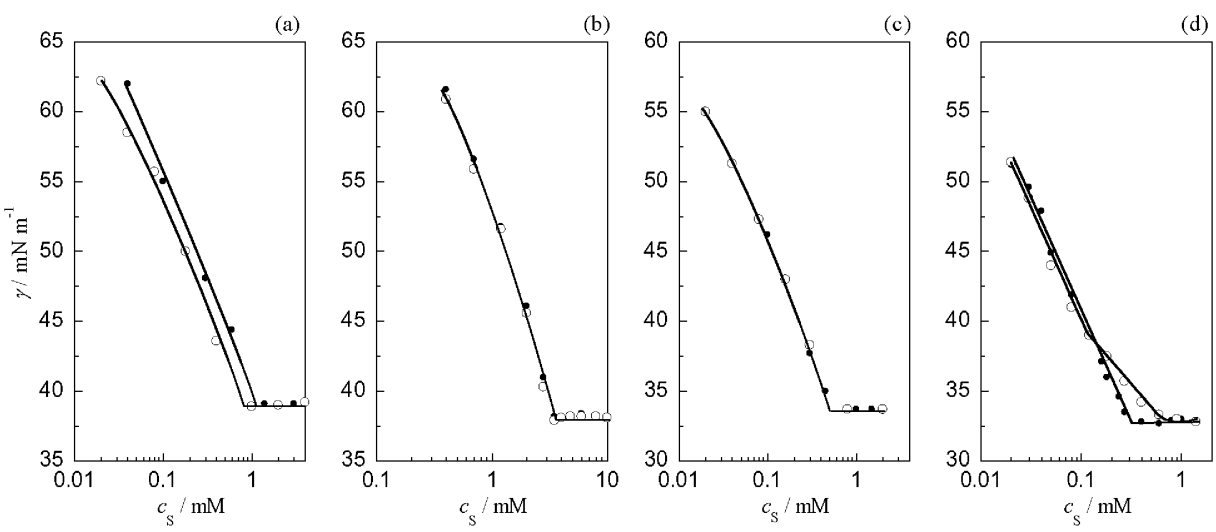

Fig.1 Surface Tension vs Concentration Curves for Various Surfactants in $0.1 \mathrm{M}$ tris- $\mathrm{HCl}$ Buffer Solution at $298.2 \mathrm{~K}$. Open circles: with $0.1 \mathrm{mM}$ of DPPC, filled circles: without vesicle. (a) SDS, (b) C12Gly, (c) C12Leu, (d) C12Phe. 
(a)
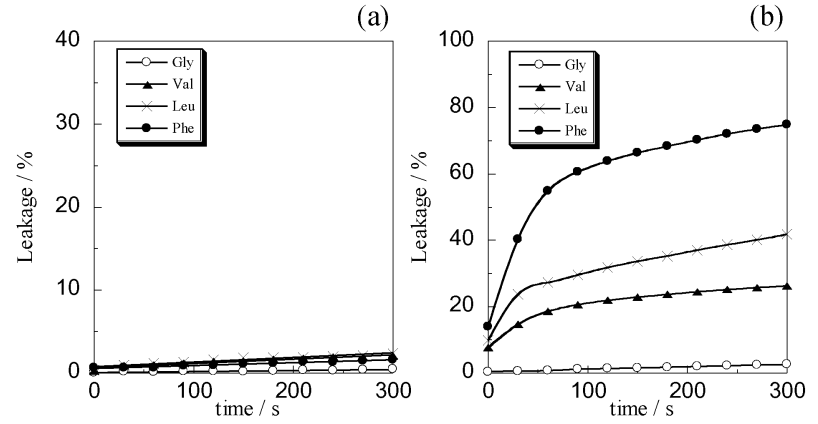

(c)

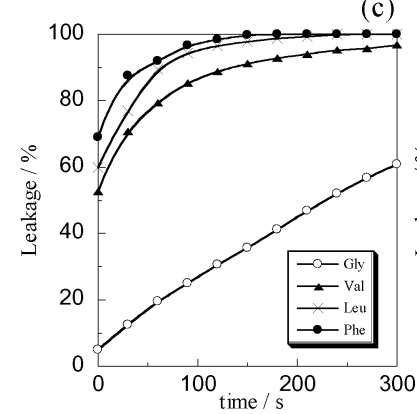

Fig.2 Leakage Ratio of CF vs Time for DPPC Vesicles in Each Surfactant Solution.

The reduced surfactant concentration, $C^{*}=c_{\mathrm{s}} / \mathrm{CMC}$, is (a) 0.02 , (b) 0.2 , (c) 1 and (d) 4 .

released mostly below the $\mathrm{CMC}$ for all systems. In particular for the hydrophobic amino acid systems, the leakage ratio reached $100 \%$ even at the concentration lower than the CMC. These results suggest that the solubilization of liposome is not the only mechanism of dye leakage induced by amino acid surfactants. In the previous study, we have reported that several amino acid type surfactants interact with DPPC vesicles cooperatively to form pore structure in the vesicle membrane, which is large enough for $\mathrm{CF}$ molecules to pass through the membrane ${ }^{27,34)}$. Karatekin et al. have reported the toroidal membrane pore formed by surfactant molecules ${ }^{35,36)}$. The CF molecules might pass through the pore consisting of amino acid type surfactants.

Next, we examined the effect of surfactants on membrane fluidity by measuring the polarization of DPH embedded in DPPC membrane. The polarization in the presence of surfactant, $P$, was divided by that in surfactant-free sample, $P_{0}(=0.42 \pm 0.03)$ to obtain the relative polarization, $P / P_{0}$. Figure 4 shows the surfactant concentration dependence of $P / P_{0}$ values measured at $2 \mathrm{~h}$ after the addition of surfactant. The $P$ values did not change with time in contrast to the dye leakage (Data not shown). It is seen that below the CMCs, the $P / P_{0}$ values hardly change from that in surfactant-free solution regardless of a variation of the surfactant concentration except for the $\mathrm{C} 12 \mathrm{Phe}$ system. It is interesting that the $P / P_{0}$ values around $C^{*}=0.5$ are still kept at unity whereas the encapsu-
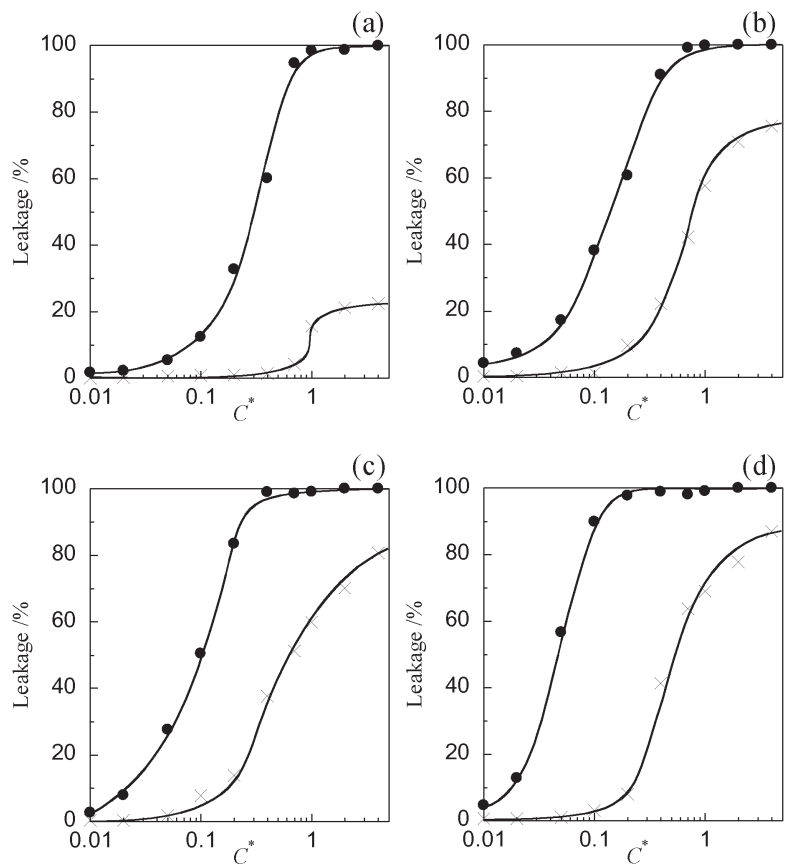

Fig.3 CF Leakage Ratio vs Reduced Surfactant Concentration Curves for DPPC Vesicles in Each Surfactant Solution at 298.2 K.

Crosses: just after mixing, filled circles: at two hours after mixing.

(a) C12Gly, (b) C12 Val, (c) C12Leu, (d) C12Phe.

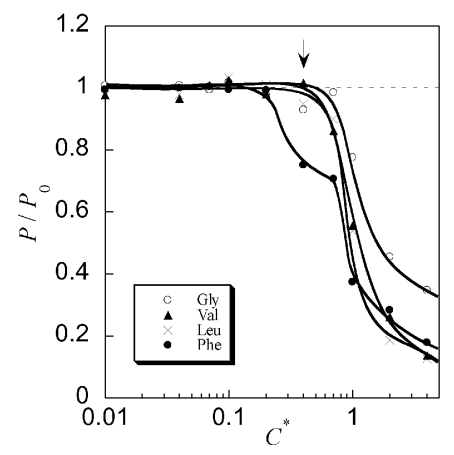

Fig.4 Polarization Ratio vs Reduced Surfactant Concentration Curves for DPPC Vesicle Membrane in Each Surfactant Solution Obtained at Two Hours after Mixing at $298.2 \mathrm{~K}$.

Inserted arrow indicates $C^{*}=0.5$.

lated probe molecules in these vesicles are almost released under the same condition. This fact supports the above idea that these amino acid-type surfactants are localized to oligomeric domain structure. Therefore, it is hard for the probe molecules to interact with surfactant molecules in lipid membrane. Above the $\mathrm{CMC}$, the $P / P_{0}$ values steeply decreased because of the solubilization of probe molecules 
into mixed micelles of DPPC and surfactant, which have much higher fluidity than vesicles. It is noted that the $P / P_{0}$ value of $\mathrm{C} 12 \mathrm{Phe}$ system exhibits a two-step decrease with increasing concentration. This unique trend was also observed in the surface tension measurement as described above. This result suggests that $\mathrm{C} 12 \mathrm{Phe}-\mathrm{DPPC}$ complex forms a different phase just below the CMC. Although the structure of this phase is not clear at the present time, the lipid membrane in this phase displays higher fluidity and the significant leakage of $\mathrm{CF}$ in this concentration region.

\subsection{Calorimetry}

In order to clarify the interaction between DPPC vesicle and the amino acid surfactants, the heat of titration of their micellar solutions to some vesicle suspensions of different concentrations was measured by ITC at $298.15 \mathrm{~K}$. The differential enthalpy $\Delta h_{\mathrm{S}}$, which was obtained by dividing the titration heat by the number of moles of injected solute, was plotted against the concentration of surfactant (Fig.5). The result of sodium dodecyl sulfate (SDS) system was shown in Fig.5(a) for comparison. The calorimetric examination for C12Val system was omitted in this study, since it is expected from the fluorescence results that C12Val system bears a striking resemblance to C12Leu system.

Typical sigmoidal curves were obtained for all systems without DPPC, and then we obtained the value of enthalpy of micelle formation from these curves ${ }^{28}$. The values of enthalpy of micelle formation are positive for C12Gly and C12Leu systems, while they are negative for $\mathrm{C} 12 \mathrm{Phe}$ and SDS systems. It has been found that the dehydration of the surfactant usually plays an important role in micelle formation of $N$-acyl amino acid surfactant ${ }^{28)}$, which contributes to positive enthalpy change. For the case of micelle forma- tion of $\mathrm{C} 12 \mathrm{Phe}$, however, the attractive interaction between the benzyl groups of side chain of C12Phe would bring about a decrease of the enthalpy change upon micellization, resulting in the negative enthalpy of micelle formation. It is seen that the differential enthalpy value does not depend on the DPPC concentration so much at lower surfactant concentration for the amino-acid surfactant systems. For the SDS system, on the other hand, the enthalpy values deviated from the curve for the DPPC-free solution at very low concentration. This result supports our previous finding that the SDS molecules were adsorbed homogeneously on DPPC vesicles even at very low concentrations below $C^{*}=0.1$, while the amino acid surfactants hardly affect the property of membrane in the low concentration range ${ }^{27}$. However when the surfactant concentration approached to the $\mathrm{CMC}$, the endothermic peak appeared on the differential enthalpy vs concentration curve, and then the enthalpy value of the peak top increased with increasing DPPC concentration for all surfactant systems. This implies that the large enthalpy is necessary for the incorporation of surfactant into the vesicles. It is suggested, furthermore, that the incorporation of surfactant reaches maximum at the peak top concentration, and the mixed micelles begin to form beyond the concentration. Therefore the concentrations at the peak top of these curves were considered as the $\mathrm{CMC}$ of the surfactants in the presence of DPPC vesicles. These results are consistent with those obtained by the surface tension measurements. That is, the addition of the DPPC vesicles brought about an increase of $\mathrm{CMC}$ in $\mathrm{C} 12 \mathrm{Phe}$ system and a decrease in SDS system, and did not give a significant effect on the CMCs of C12Gly and C12Leu.

If we can neglect the changes in concentrations of solvent and DPPC during the titration, the differential
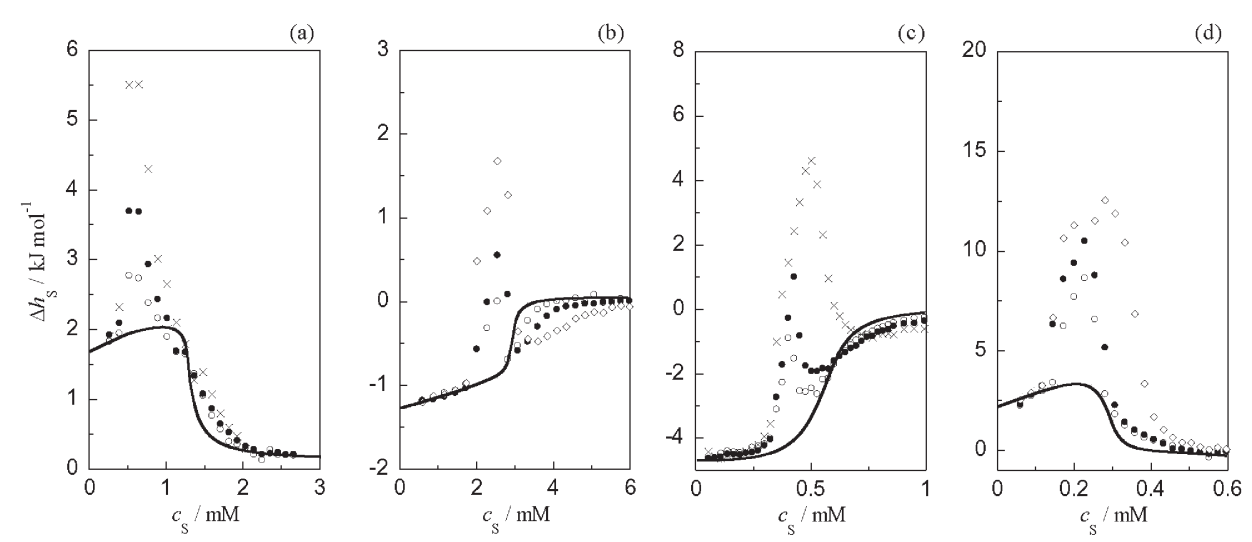

Fig.5 Titration Heat per Mole of Surfactant vs Concentration Curves with/without DPPC at $298.15 \mathrm{~K}$.

Open circles : $c_{\mathrm{L}}=0.023 \mathrm{mM}$, filled circles: $c_{\mathrm{L}}=0.046 \mathrm{mM}$, diamonds: $c_{\mathrm{L}}=$ $0.092 \mathrm{mM}$, crosses: $c_{\mathrm{L}}=0.138 \mathrm{mM}$, solid line: without DPPC.

(a) SDS, (b) C12Gly, (c) C12Leu, (d) C12Phe. 
enthalpy $\Delta h_{\mathrm{S}}$ is regarded as the partial derivative of enthalpy of mixing $H^{\mathrm{M}}$ with respect to moles of surfactant $n_{\mathrm{S}}$, and is expressed as following equation,

$$
\Delta h_{s} \approx\left(\partial H^{M} / \partial n_{s}\right)_{T, p, n i \neq s}=h_{s}-h_{s}^{*}
$$

where $h_{\mathrm{S}}$ is the partial molar enthalpy of surfactant in the solution after mixing and $h_{S}^{*}$ is that in the titrant solution of surfactant, respectively. Now let us consider the two states for the surfactant molecules; one is the free monomer in solution and the other is the bound one to the DPPC vesicles. Then eq.[5] is changed as

$$
\Delta h_{s} \approx\left(\partial c_{s}^{f} / \partial c_{s}\right)_{T, p}\left(h_{s}^{f}-h_{s}^{*}\right)+\left(\partial c_{s}^{b} / \partial c_{s}\right)_{T, p}\left(h_{s}^{b}-h_{s}^{*}\right)[6]
$$

Here $c_{\mathrm{S}}$ is molarity of surfactant, and the superscripts " $\mathrm{f}$ " and "b" refer to the free monomer state in solution and the bound state to the DPPC vesicles, respectively. The total molarity of the surfactant is given by the mass balance relation:

$$
c_{\mathrm{S}}=c_{\mathrm{s}}^{\mathrm{f}}+c_{\mathrm{s}}^{\mathrm{b}} .
$$

Next we consider the chemical equilibrium between the surfactant monomer in solution and that bound to vesicles. The binding constant of surfactant to vesicles is defined by

$$
K=\frac{c_{\mathrm{S}}^{\mathrm{b}}}{c_{\mathrm{L}} v_{\mathrm{L}} c_{\mathrm{s}}^{\mathrm{f}}} .
$$

Here $c_{\mathrm{L}}$ and $v_{\mathrm{L}}$ are molarity and molar volume of DPPC. Substitution of eqs. [7] and [8] into eq. [6] yields the expression:

$$
\Delta h_{s}=\frac{K v_{\mathrm{L}} c_{\mathrm{L}}}{1+K v_{\mathrm{L}} c_{\mathrm{L}}} \Delta h_{\mathrm{S}}^{\mathrm{b}}+\Delta h_{\mathrm{s}}^{\mathrm{f}},
$$

where the binding enthalpy of surfactant $\Delta h_{\mathrm{S}}^{\mathrm{b}}$ and the enthalpy of dilution of surfactant $\Delta h_{\mathrm{S}}^{\mathrm{f}}$ are defined as

$$
\Delta h_{\mathrm{s}}^{\mathrm{b}}=h_{\mathrm{s}}^{\mathrm{b}}-h_{\mathrm{s}}^{\mathrm{f}}
$$

and

$$
\Delta h_{\mathrm{s}}^{\mathrm{f}}=h_{\mathrm{s}}^{\mathrm{f}}-h_{\mathrm{s}}^{*},
$$

respectively. It was assumed that the molar volume of DPPC was independent of surfactant concentration.

The maximum enthalpy values of each plot in Fig. 5 were plotted against $c_{\mathrm{L}}$ in Fig.6 for all surfactant systems, and eq. [9] was applied to data points for further analysis. The $\Delta h_{S}^{\mathrm{f}}$ values, which are roughly equal to the enthalpy of demicellization, can be obtained by the titration of surfactant solution without vesicles. Thus, the fitting of the experimental data as a function of $c_{\mathrm{L}}$ to Eq. [9] derives both the binding constant and the binding enthalpy. It is seen that the fitting curves reproduce the experimental results very well. Here $v_{\mathrm{L}}$ was estimated as $0.91 \mathrm{dm}^{3} \mathrm{~mol}^{-1}$ by the use of experimental data of molecular area $a_{\mathrm{L}}\left(=0.41 \mathrm{~nm}^{2)}\right.$ and bilayer pitch $d_{\mathrm{L}}(=7.4 \mathrm{~nm})$ of hydrated DPPC in gel phase ${ }^{37,38)}$. Obtained results were summarized in Table 1. It is seen that the $\Delta h_{\mathrm{S}}^{\mathrm{b}}$ value is positive and is hardly affected by a type of surfactant. The increase of enthalpy would be mainly due to a decrease of interaction between acyl chains of DPPC molecules in the vesicle membrane, which is caused by the insertion of the surfactant hydrocarbon chains into the membrane. This interpretation is consistent with the fact that the binding enthalpy is independent of the surfactant head group, but is determined by a hydrocarbon part of the surfactants.

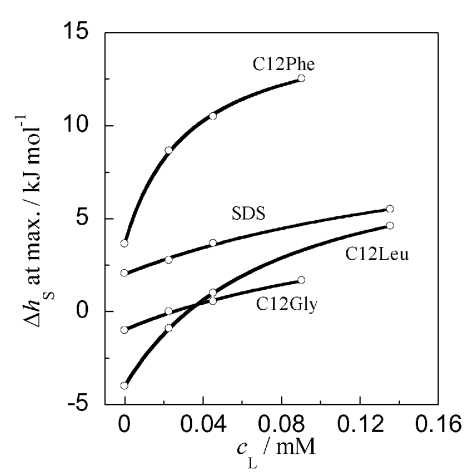

Fig.6 Maximum Enthalpy vs DPPC Concentration Curves for Each Surfactant System. Solid lines are the fitting results by eq.[9] .

Table 1 Calculated Thermodynamic Parameters by ITC.

\begin{tabular}{cccccc}
\hline surfactant & $K \times 10^{3}$ & $R$ & $\begin{array}{c}\Delta h_{\mathrm{s}}^{\mathrm{b}} / \\
\mathrm{kJ} \mathrm{mol}^{-1}\end{array}$ & $\begin{array}{c}\Delta h_{\mathrm{s}}^{\mathrm{f}} / \\
\mathrm{kJ} \mathrm{mol}^{-1}\end{array}$ & $c_{\mathrm{s}}^{\mathrm{f}} / \mathrm{mM}$ \\
\hline C12Gly & $2.3 \pm 1.1$ & 4.6 & $13.5 \pm 3.0$ & -0.88 & 2.2 \\
C12Leu & $12 \pm 0.1$ & 3.1 & $13.4 \pm 0.1$ & -4.0 & 0.28 \\
C12Phe & $28 \pm 0.2$ & 2.9 & $12.0 \pm 0.4$ & 3.6 & 0.12 \\
SDS & $2.7 \pm 1.5$ & 1.0 & $12.0 \pm 5.0$ & 2.0 & 0.43 \\
\hline
\end{tabular}


On the other hand, the binding constant depends strongly on a kind of surfactant. Especially the amino acid surfactant with a hydrophobic side chain has a large value of $K$. This result suggests that an increase of entropy strongly dominates the binding process. Moreover, the hydrophobic effect of amino acid side chain is also one of the key factors for the binding of surfactant to lipid membrane.

To examine the binding of the surfactants to DPPC vesicles in more detail, we estimated the surfactant to DPPC molar ratio, $R$, in the vesicle membrane saturated with the surfactant molecules as follows:

$$
R=\frac{c_{\mathrm{s}}^{\mathrm{b}}}{c_{\mathrm{L}}}=K v_{\mathrm{L}} c_{\mathrm{s}}^{\mathrm{f}} .
$$

In this study, the concentration of the free monomer of surfactant $c_{S}^{f}$ was calculated by eqs. [7] and [8] under the condition of $c_{\mathrm{L}}=0.046 \mathrm{mM}$. Total concentration $c_{\mathrm{S}}$ in eq. [7] was considered as the concentration at which the differential enthalpy $\Delta h_{\mathrm{S}}$ was maximum in Fig.5. Obtained data was also shown in Table 1. It is seen that the $R$ values of these amino acid surfactant systems are greater than unity and increase with decreasing size of amino acid side chain. Although a number of surfactant molecules bound to the DPPC vesicle membrane, the environment around DPPC in the vesicle was not changed so much by the adsorption as demonstrated by polarization result. This result supports our depiction, in which the amino acid-type surfactant molecules are organized in the DPPC vesicle membrane ${ }^{27,34)}$. On the other hand, $R$ value of SDS system is almost unity, though SDS molecules affect the DPPC vesicle at even lower reduced concentration compared with the amino acid-type surfactant systems. It was confirmed again that this surfactant adsorbed homogeneously on DPPC vesicles.

\section{CONCLUSION}

The incorporation behaviors of four $N$-dodecanoyl amino acid-type surfactants to DPPC membrane in aqueous solution were investigated by fluorescence probe method and ITC. These surfactants did not leak the encapsulated probes in the DPPC vesicle so much in a low concentration range, but caused an immediate release of the probe from the vesicles at the concentration around the $\mathrm{CMC}$. The leakage induced by amino acid-type surfactant was promoted by increasing hydrophobicity of the amino acid. The encapsulated probe molecules were released completely by incorporation of these surfactants even at the concentration below the CMC, whereas the polarization of the DPPC membrane was still high at the corresponding concentration. In addition, in both the surface tension and DPH measurement, $\mathrm{C} 12 \mathrm{Phe}$ displayed different trend from other surfactants. The binding enthalpy and the binding constant of the surfactant to DPPC vesicles were estimated by analyzing the ITC results thermodynamically. The binding enthalpy was almost independent of a type of surfactants; on the other hand, the binding constant increases with increasing hydrophobicity of the surfactant. The molar ratio of adsorbed surfactant to DPPC molecule in the saturated state for amino acid-type surfactant was much greater than that for sodium dodecyl sulfate system. The difference in the incorporation behavior between the amino acid-type surfactants and SDS can be discussed from the viewpoint of cooperative adsorption. That is, the amino acid-type surfactants might be incorporated into the DPPC vesicle cooperatively, and then, be organized on the membrane.

\section{ACKNOWLEDGEMENT}

The authors are also grateful to Prof. Nobuhiko Yui and Dr. Ryo Katoono of the Japan Advanced Institute of Science and Technology for the ITC experiment. This work was partly supported by the Cosmetology Research Foundation and the Japan Society for the Promotion of Science (Grant in Aid for Young Scientists [B] No. 20750102).

\section{References}

1. Heerklotz, H. Interactions of surfactants with lipid membranes. Quart. Rev. Biophys. 41, 205-264 (2008).

2. Koynova, R.; Tenchov, B. Interactions of surfactants and fatty acids with lipids. Curr. Opin. Colloid Interface Sci. 6, 277-286 (2001).

3. Le Maire, M.; Champeil, P.; Møller, J.V. Interaction of membrane proteins and lipids with solubilizing detergents. Biochim. Biophys. Acta. 1508, 86-111 (2000).

4. Lopez, O.; De La Maza, A.; Coderch, L.; Parra, J.L. Selective solubilization of the stratum corneum components using surfactants. Prog. Colloid Polym.Sci. 100, 230-234 (1996).

5. Dennis, E.A. Micellization and solubilization of phospholipids by surfactants. Adv. Colloid Interface Sci. 26, 155-175 (1986).

6. Womack, M.D.; Kendall, D.A.; MacDonald, R.C. Detergent effects on enzyme activity and solubilization of lipid bilayer membranes. Biochim. Biophys. Acta. 733, 210-215 (1983).

7. Carmona-Ribeiro, A.M. Lipid bilayer fragments and disks in drug delivery. Curr. Med. Chem. 13, 13591370 (2006).

8. Barriocanal, L.; Taylor, K.M.G.; Buckton, G. Bilayer to micelle transition of DMPC and alcohol ethoxylate surfactants as studied by isoperibol calorimetry. J. Parm. Sci. 94, 1747-1755 (2005).

9. Lim, W.H.; Lawrence, M.J. Influence of surfactant and 
lipid chain length on the solubilisation of phosphatidylcholine vesicles by micelles comprised of polyoxyethylene sorbitan monoesters. Colloids Surf. A 250, 449457 (2004).

10. Patra, S.K.; Alonso, A.; Goñi, F.M. Detergent solubilisation of phospholipid bilayers in the gel state: The role of polar and hydrophobic forces. Biochim. Biophys. Acta. 1373, 112-118 (1998).

11. Patra, S.K.; Alonso, A.; Goñi, F.M. Detergent solubilisation of phospholipid bilayers in the gel state: The role of polar and hydrophobic forces. Biochim. Biophys. Acta. 1373, 112-118 (1998).

12. De La Maza, A.; Parra, J.L. Solubilizing effects caused by the nonionic surfactant dodecylmaltoside in phosphatidylcholine liposomes. Biophys. J. 72, 1668-1675 (1997).

13. De La Maza, A.; Parra, J.L. Assembly properties of Triton X-100/phosphatidylcholine aggregates during liposome solubilization. Colloid Polym. Sci. 274, 866-874 (1996).

14. Partearroyo, M.A.; Alonso, A.; Goñi, F.M.; Tribout, M.; Paredes, S. Solubilization of phospholipid bilayers by surfactants belonging to the Triton X series: Effect of polar group size. J. Colloid Interface Sci. 178, 156-159 (1996).

15. De La Maza, A.; Parra, J.L. Solubilization of unilamellar phospholipid bilayers by nonionic surfactants. Colloid Polym. Sci. 272, 721-730 (1994).

16. Inoue, T.; Kawamura, H.; Okukado, S.; Shimozawa, R. Characterization of molecular assemblies formed in aqueous C10E7/DPPC mixture by spin label and fluorescence probe techniques and mechanism of micelleto-vesicle transformation. J. Colloid Interface Sci. 168, 94-102 (1994).

17. Møller, J.V. Binding of a nonionic detergent to membranes: Flip-flop rate and location on the bilayer. Biochemistry 26, 4803-4810 (1987).

18. Deo, N.; Somasundaran, P. Effects of sodium dodecyl sulfate on mixed liposome solubilization. Langmuir 19, 7271-7275 (2003).

19. Deo, N.; Somasundaran, P. Disintegration of liposomes by surfactants: Mechanism of protein and cholesterol effects. Langmuir 19, 2007-2012 (2003).

20. Lopez, O.; Cocéra, M.; Parra, J.L.; Coderch, L.; De La Maza, A. Influence of ceramides in the solubilization of stratum corneum lipid liposomes by C12-betaine/sodium dodecyl sulfate mixtures. Int. J. Pharm. 187, 231241 (1999).

21. De La Maza, A.; Parra, J.L. Intermediate aggregates resulting in the interaction of sodium dodecyl sulphate with phosphatidylcholine liposomes. Colloids Surf. A 112, 63-71 (1996).

22. Infante, M.R.; Pérez, L.; Pinazo, A.; Clapés, P.; Morán, M.C.; Angelet, M.; García, M.T; Vinardell, M.P. Amino acid-based surfactants. Comptes Rendus Chimie 7, 583-592 (2004).

23. Takehara, M. Properties and applications of amino acid based surfactants. Colloids Surf . 38, 149-167 (1989).

24. Castillo, J.A; Pinazo, A; Carilla, J; Infante, M.R; Alsina, M.A.; Haro, I; Clapés, P. Interaction of antimicrobial arginine-based cationic surfactants with liposomes and lipid monolayers. Langmuir 20, 3379-3387 (2004).

25. Epand, R.F.; Infante, M.R.; Flanagan, T.D.; Epand, R.M. Properties of lipoamino acids incorporated into membrane bilayers. Biochim. Biophys. Acta 1373, 67-75 (1998).

26. Kondoh, M.; Furutani, T.; Azuma, M.; Ooshima, H.; Kato, J. Acyl amino acid derivatives as novel inhibitors of influenza neuraminidase. Biosci. Biotech. Biochem. 61, 870-874 (1997).

27. Yasuhara, K.; Ohta, A.; Asakura, Y.; Kodama, T.; Asakawa, T.; Miyagishi, S. Unique incorporation behavior of amino acid-type surfactant into phospholipid vesicle membrane. Colloid Polym. Sci. 283, 987993 (2005).

28. Ohta, A.; Toda, K.; Morimoto, Y.; Asakawa, T.; Miyagishi, S. Effect of the side chain of $\mathrm{N}$-acyl amino acid surfactants on micelle formation: An isothermal titration calorimetry study. Colloids Surf. A 317, 316-322 (2008).

29. Ohta, A.; Ozawa, N.; Nakashima, S.; Asakawa, T.; Miyagishi, S. Krafft temperature and enthalpy of solution of $\mathrm{N}$-acyl amino acid surfactants and their racemic modifications: Effect of the amino acid residue. Colloid Polym. Sci. 281, 363-369 (2003).

30. Frisken, B.J.; Asman, C.; Patty, P.J. Studies of vesicle extrusion. Langmuir 16, 928-933 (2000).

31. Takayama, M.; Itoh, S.; Nagasaki, T.; Tanimizu, I. A new enzymatic method for determination of serum choline containing phospholipids. Clin. Chim. Acta 79, 93-98 (1977).

32. Lentz, B.R. Membrane 'efluidity' as detected by diphenylhexatriene probes. Chem. Phys. Lipids 50, 171-190 (1989).

33. Can, S.Z.; Chang C.F.; Walker, R.A. Spontaneous formation of DPPC monolayers at aqueous/vapor interfaces and the impact of charged surfactants. Biochim. Biophys. Acta 1778, 2368-2377 (2008).

34. Yasuhara, K.; Shimanouchi, T.; Umakoshi, H.; Kuboi, Fluorescence study on the domain formation of Ndodecanoyl -L-tryptophan within a liposome membrane. Colloid Polym. Sci. 285, 239-243 (2006).

35. Karatekin, E.; Sandre, O.; Brochard-Wyart, F. Transient pores in vesicles. Polym. Intl. 52, 486-493 (2003).

36. Puech, P.-H.; Borghi, N.; Karatekin, E.; BrochardWyart, F. Line thermodynamics: Adsorption at a membrane edge. Phys. Rev. Lett. 90, 128304/1-128304/4 (2003). 
37. Braganza, L.F. Hydrostatic pressure induces hydrocarbon chain interdigitation in single-component phospholipid bilayers. Biochem. 25, 2591-2596 (1986).

38. Ruocco, M.J.; Graham Shipley, G. Characterization of the sub-transition of hydrated dipalmitoylphosphatidylcholine bilayers. X-ray diffraction study. Biochim. Biophys. Acta 684, 59-66 (1982). 